\title{
Evaluation of processed bovine cancellous bone matrix seeded with syngenic osteoblasts in a critical size calvarial defect rat model
}

\author{
U. Kneser a, L. Stangenberg b, J. Ohnolz a, b, O. Buettner b, J. Stern-Straeter b, \\ D. Möbest b, R. E. Horch a, *, G. B. Stark b, D. J. Schaefer c \\ a Department of Plastic and Hand Surgery, University of Erlangen Medical Center, Erlangen, Germany \\ ${ }^{b}$ Department of Plastic and Hand Surgery, University of Freiburg Medical Center, Freiburg, Germany \\ c Department of Plastic, Reconstructive and Aesthetic Surgery, Clinic of Reconstructive Surgery, \\ University Hospital, Basel, Switzerland
}

Received: March 23, 2006; Accepted: June 20, 2006

\begin{abstract}
Introduction: Biologic bone substitutes may offer alternatives to bone grafting procedures. The aim of this study was to evaluate a preformed bone substitute based on processed bovine cancellous bone (PBCB) with or without osteogenic cells in a critical size calvarial defect rat model. Methods: Discs of PBCB (Tutobone ${ }^{\circledR}$ ) were seeded with second passage fibrin gel-immobilized syngenic osteoblasts (group A, $n=40$ ). Cell-free matrices (group $B, n=28$ ) and untreated defects (group $\mathrm{C} ; \mathrm{n}=28$ ) served as controls. Specimens were explanted between day 0 and 4 months after implantation and were subjected to histological and morphometric evaluation. Results: At 1 month, bone formation was limited to small peripheral areas. At 2 and 4 months, significant bone formation, matrix resorption as well as integration of the implants was evident in groups A and B. In group C no significant regeneration of the defects was observed. Morphometric analysis did not disclose differences in bone formation in matrices from groups A and B. Carboxyfluorescine-Diacetate-Succinimidylester (CFDA) labeling demonstrated low survival rates of transplanted cells. Discussion: Osteoblasts seeded into PBCB matrix display a differentiated phenotype following a 14 days cell culture period. Lack of initial vascularization may explain the absence of added osteogenicity in constructs from group A in comparison to group B. PBCB is well integrated and represents even without osteogenic cells a promising biomaterial for reconstruction of critical size calvarial bone defects.
\end{abstract}

Keywords: bone tissue engineering - osteoconduction - osteointegration - osteoblast transplantation processed bovine cancellous bone matrix

\section{Introduction}

\section{Autologous bone grafts}

Currently, autologous bone grafting is the gold standard for osteogenic bone replacement. This proce-

\footnotetext{
* Correspondence to: Raymund E. HORCH, M.D. Department of Plastic and Hand Surgery, University of Erlangen Medical Center, Krankenhausstrasse 12, 91054 Erlangen, Germany.

Tel.: +49-9131-85-33277

Fax: +49-9131-85-39327

E-mail: raymund.horch@chir.imed.uni-erlangen.de
}

dure is highly efficient in reconstructing bone defects even under problematic conditions. However, the use of autologous bone grafts is limited by a significant donor site morbidity that increases with the amount of bone harvested [1].

\section{Biomaterials for bone replacement}

A plethora of different types of acellular biomaterials has been developed for bone replacement as 
alternatives to autologous bone grafts [2-4]. Besides injectable or moldable materials $[5,6]$, solid scaffolds are widely used in experimental and clinical settings, when maintenance of shape or high initial stability are required. Ideally, biomaterials for bone replacement are biocompatible, osteoconductive (i.e. guidance of bone ingrowth from the surrounding bone into the biomaterial), non-immunogenic, mechanically stable and slowly biodegradable. The scaffolds should participate in the remodeling process in order to prevent stress shielding phenomenon. Polymers and ceramics represent important groups of biomaterials for bone replacement [7-11]. Ceramics provide osteoconductivity and are easily integrated into bone defects. However, in contrast to polymers, many ceramics are brittle and lack tensile strength. This tends to limit their use in applications that require significant resistance against torsion, bending, or shear stress.

Processed acellular cancellous bone, either of allogenic or xenogenic origin, represents another type of biomaterial for bone replacement. This biogenic material has many beneficial properties for bone replacement: generally spoken its micro- and macro architecture perfectly matches with the recipient's bone. Following adequate processing, the chemical composition regarding mineral content resembles the one of native bone. Processed bone implants have high compressive strength and elasticity and tolerate shear stress very well [12, 13]. They are available in nearly unlimited quantities. However, the processing technique is of crucial importance for the in vitro and in vivo performance of allogenic or xenogenic bone implants. Adequate processing has to guarantee removal of donor cells, elimination of cytotoxic agents, antigenicity and pathogens from the implant [14]. At the same time it ideally preserves the chemical composition of the bone scaffold and does not significantly impair elasticity and compressive strength, integration and osteoconduction of the implants. Adhesion, proliferation and differentiation of osteogenic cells within PBCB matrices as well as secretion of calcified extracellular matrix have been demonstrated [14-16]. Bone defects have been successfully reconstructed using allogenic processed human cancellous bone matrices [17].

\section{Enhancement of bone formation in biomaterials}

Bone formation in biomaterials proceeds from the edges of the material, which are in contact to the surrounding bone, towards the center (osteoconduction). Bone formation could further be increased by following tissue engineering strategies: Osteogenicity may be added to biomaterials for bone tissue engineering by means of incorporation of osteoblasts, mesenchymal stem cells, periosteal cells or other osteogenic cell types [8-10, 14, 18-21]. Bone formation could also be induced by immobilization of osteoinductive growth factors within the scaffolds [22-25]. Gene transfer strategies may achieve transient or permanent expression of growth factors within biomaterials for enhanced bone formation $[26,27]$.

\section{Aim of study}

In this study we investigated a processed bovine cancellous bone matrix in a critical size calvarial defect rat model. The aim of this experimental study was twofold: firstly to evaluate osseointegration and osteoconductive properties of unmodified PBCB implants. Secondly to assess the osteogenic potential of syngenic osteoblasts incorporated in PBCB matrices in comparison with cell-free PBCB implants.

\section{Materials and methods}

\section{Experimental design}

Implants were investigated in vivo in a standardized calvarial defect rat model. According to Schmitz et al., the critical defect size that does not heal spontaneously is 8 $\mathrm{mm}$ in the rat calvarial defect model [28]. The study design is outlined in Table 1. Briefly, standardized critical size calvarial defects were generated in 101 WistarKyoto rats. Only animals that survived the first $24 \mathrm{~h}$ following surgery $(\mathrm{n}=96)$ were included into the study. Experimental animals were divided into 3 groups. Defects in group A $(n=40)$ were filled with PBCB matrices seeded with fibrin gel-immobilized primary osteoblasts. Animals from group B $(\mathrm{n}=28)$ received cell-free matrices. Defects in group C $(=28)$ were left 
Table 1 Experimental design

\begin{tabular}{|c|c|c|c|c|c|c|c|c|}
\hline & day 0 & day 1 & day 3 & day 7 & 1 month & 2 months & 4 months & excluded \\
\hline $\begin{array}{l}\text { group A } \\
(\mathrm{PBCB}+\mathrm{OB})\end{array}$ & 4 & 4 & 4 & 4 & 8 & 8 & 8 & (2) \\
\hline group B (PBCB) & 4 & 0 & 0 & 0 & 8 & 8 & 8 & (2) \\
\hline $\begin{array}{l}\text { group C } \\
\text { (control) }\end{array}$ & 4 & 0 & 0 & 0 & 8 & 8 & 8 & (1) \\
\hline
\end{tabular}

$\mathrm{PBCB}=$ processed bovine cancellous bone matrix, $\mathrm{OB}=$ osteoblasts. Numbers of experimental animals included in the study are given for each group and time point following implantation. Numbers of animals excluded are given in parentheses.

untreated and served as control. At day 0, 4 specimens per group and time were harvested. Between days 1 and 7, 12 constructs from group A containing CarboxyFluorescine Diacetate-succinimidyl ester (CFDA) labeled cells were subjected to histological evaluation for assessment of initial cell survival. At 1, 2, and 4 months following induction of the defects, 8 specimens per group and time were harvested and analyzed. In addition to the in vivo experiments 4 cell-seeded matrices were evaluated in vitro in $3 \mathrm{D}$ culture. These matrices were investigated at day 14 (time point of implantation in group A) and subjected to histological evaluation as described below.

\section{Animal model and surgical procedures}

Syngenic male Wistar-Kyoto rats (10 weeks old, 240-280 g; Charles River Laboratories, Sulzfeld, Germany) served as donors and recipients. German regulations for the care and use of laboratory animals were observed at all times. All experiments were approved by the animal care committee of the University of Freiburg. The animals were housed in the veterinary care facility of the University of Freiburg Medical Center and submitted to a $12 \mathrm{~h}$ dark/light cycle with free access to standard chow (Altromin, Hamburg, Germany) and water.

At the commencement of surgery, all rats were given $0.2 \mathrm{ml}$ of a broad spectrum depot antibiotic (Tardomycel Comp III, Bayer, Leverkusen, Germany). Animals were anesthetized with an intramuscular injection of ketamin (100 mg/kg, Essex Pharma, Munich, Germany) and xylazin ( $5 \mathrm{mg} / \mathrm{kg}$, Bayer, Leverkusen, Germany). The surgical site was shaved, prepped and draped for sterili- ty. Trephination began with a midline cranial incision extending from the posterior occipital protuberance to the nasal bone. A $7.9 \mathrm{~mm}$ trephine was used to create a midline parietal craniotomy. Care was taken to preserve the dura, the sagittal sinus and the underlying brain. In animals from groups A and B, processed bovine cancellous bone discs were implanted in a press fit fashion. No osteofixation device was used. Cell-seeded implants for animals in group A were consequently kept on ice prior to implantation. Animals from group $\mathrm{C}$ did not receive any matrix and served as negative control. Hemostasis was assured and the wound was closed in two layers using Vicryl 5-0 (Ethicon, Norderstedt, Germany).

\section{Isolation and expansion of primary rat osteoblasts}

After sacrificing the rats by $\mathrm{CO}_{2}$ asphyxiation, femora, tibiae and humeri were dissected and transferred into Dulbecco's Modified Eagle Medium (DMEM, Life Technologies, Paisley, UK), containing Penicillin/Streptomycin $100 \mathrm{U} / \mathrm{ml}$ (Life Technologies). In the cell culture laboratory, samples were cleaned of from soft tissue and cartilage and minced into $2 \mathrm{~mm}$ pieces. $20 \mathrm{ml}$ Collagenase type II solution $(2 \mathrm{mg} / \mathrm{ml} \mathrm{col}-$ lagenase type 2 (Biochrom, Berlin, Germany)) in DMEM were added. Bone pieces were digested for 30 min at $37^{\circ} \mathrm{C}$ under continuous mechanic agitation. The supernatant from the first digestion step was discarded. Digestion was then repeated three times. The supernatant was pooled and purified by centrifugation (10 $\mathrm{min}, 1200$ $\left.\mathrm{rpm}, 4^{\circ} \mathrm{C}\right)$. Cell pellets were resuspended in basal medium (BM) (DMEM containing Penicillin/Streptomycin 
$100 \mathrm{U} / \mathrm{ml}$ and $10 \%$ fetal calf serum (FCS) (Biochrom) from selected lots). Cells were plated at a density of 5000 cells $/ \mathrm{cm}^{2}$ in $225 \mathrm{~cm}^{2}$ culture flasks (BD Falcon, Heidelberg, Germany) and cultured in an incubator in a humidified, $5 \% \mathrm{CO}_{2}$ atmosphere at $37^{\circ} \mathrm{C}$. Culture medium (BM) was changed every third day. Cells were passaged at $90 \%$ confluency using trypsin/EDTA (Viralex, Gibco) according to standard protocols. Second passage cells were seeded into the matrices.

\section{Seeding of processed bovine cancellous bone matrices and $3 \mathrm{D}$ culture}

Disks of processed bovine cancellous bone (diameter $8 \mathrm{~mm}$, thickness $1 \mathrm{~mm}$ ) were provided by Tutogen Medical (Neunkirchen, Germany). The bone discs were processed according to standardized protocols. According to the manufacturer this process reliably eliminates any potential pathogen from the biomaterial. Briefly, fat and surrounding connective tissue was macroscopically removed upon harvesting of the tissue. Then delipidization was performed in an acetone bath using ultrasound agitation. Alternating baths in distilled water and saline ("osmotic contrast treatment") disrupt cell wall integrity and expose intracellular structures for further processing steps. A subsequent wash with $\mathrm{H}_{2} \mathrm{O}_{2}$ destroys proteins, inactivates remaining viruses and removes residual antigenicity. Solvent dehydration in a series of 7 acetone baths removes water, destroys remaining prion or viral agents and stabilizes the graft for storage at room temperature. Gamma irradiation (17.8 kGy) sterilizes the bone grafts. Prior to implantation the PBCB discs were rehydrated by incubation in $5 \mathrm{ml} \mathrm{BM}$ containing $1000 \mathrm{U} / \mathrm{ml}$ aprotinin $\left(\right.$ Trasylol ${ }^{\circledR}$, Bayer, Leverkusen, Germany) and $100 \mathrm{IE} / \mathrm{ml}$ thrombin (TissuCol ${ }^{\circledR}$, Baxter, Vienna, Austria) at $4^{\circ} \mathrm{C}$ for $12 \mathrm{hrs} .1 .2 \times 10^{6}$ second passage primary osteoblasts were resuspended in $50 \mu \mathrm{l} \mathrm{BM}$ containing $1000 \mathrm{U} / \mathrm{ml}$ aprotinin and 10 $\mathrm{mg} / \mathrm{ml}$ fibrinogen (Sigma, Irvine, UK). Disks were dried shortly and then seeded with the osteoblast-fibrinogen solution. The seeded constructs were placed into the incubator for 30 minutes. After polymerization of the fibrin gel, disks were placed in 6-well culture dishes (BD Falcon). Eight $\mathrm{ml}$ of differentiation medium (basal medium $+25 \mathrm{mg} / 1 \mathrm{~L}$-ascorbic acid 2phosphate (Sigma, Taufkirchen, Germany), $10 \mathrm{mmol} / \mathrm{l}$ $\beta$-glycerol phosphate (Sigma), $100 \mathrm{nmol} / 1$ dexamethasone (Sigma) [29]) containing $1000 \mathrm{U} / \mathrm{ml}$ aprotinin was added per well. Differentiation medium was changed every other day. Constructs were kept in culture for 14 days prior to implantation. One hour prior to implantation constructs were transferred in sterile polypropylene tubes (BD Falcon) containing cell culture medium and placed on ice.

\section{CFDA labelling}

Initial osteoblast survival and engraftment were evaluated between days 0 and 7 using CFDA (CarboxyFluorescine Diacetate-succinimidyl ester) labelling [30]. Briefly, P2 osteoblasts were detached as described above and incubated for 15 minutes with $10 \mathrm{mM}$ freshly prepared carboxy-fluorescine diacetate-succinimidyl ester (Vybrant CFDA SE Cell Tracer-Kit, Molecular Probes, Eugene, OR) reconstituted in DMSO and PBS according to the manufacturer's recommendations. After several washing and incubation steps, cells were immobilized in fibrin gel and constructs were prepared according to the afore-mentioned protocol.

\section{Polychrome fluorescent sequential labelling}

Bone formation in the implants was evaluated using fluorescent sequential labelling techniques. Animals were injected with xylenol orange (Merck, Darmstadt, Germany, $90 \mathrm{mg} / \mathrm{kg}$ body weight), calcein green (Merck, $10 \mathrm{mg} / \mathrm{kg}$ body weight) and oxytetracylcin (Intervet, Tönisvorst, Germany, $25 \mathrm{mg} / \mathrm{kg}$ body weight) subcutaneously at 3,2 and one week prior to explantation, respectively. Unstained non-decalcified plastic-embedded cross sections were evaluated using a confocal laser microscope with appropriate filter settings (Zeiss, Oberkochen, Germany). Overlay pictures were created for all three stains using Zeiss software.

\section{Histological analysis}

Specimens were explanted together with a $15 \times 15$ $\mathrm{mm}$ piece of the surrounding calvarial bone in a standardized fashion, fixed in Schaffer's solution and dehydrated in graded acetone. After embedding in methylmethacrylate (Technovit 9100, Heraeus Kulzer Wertheim/Ts, Germany), $5 \mu \mathrm{m}$ cross sections were obtained using a Leica hard tissue microtome (Leica Microsystems, Bensheim, Germany). 
Sections were stained using hematoxylin eosin, Masson-Goldner and von Kossa stains according to standard protocols. Microphotographs were taken using Axioplan microscope and Axiocam digital camera (Carl Zeiss, Oberkochen, Germany); no digital processing of the original pictures was performed at any time.

\section{Morphometric and statistical analysis}

Histomorphometric measurements were made using the Image Tool software (University of Texas Heath Science Center, TX, USA). Two standardized central cross sections per specimen and 8 specimens per group and time were analyzed. Total defect area and total area of new bone formation was measured and the ratio between total new bone area and total defect area was calculated for each individual cross section. Values are given as mean value \pm standard deviation. Statistical analysis was performed using GraphPad Prism and SPSS software. Twotailed unpaired Student's t-test and ANOVA in combination with Tukey's Studentized Range (HSD) Test were applied for statistical analysis. The critical level of statistical significance chosen was $p<0.05$.

\section{Results}

\section{Preparation of the constructs and in vitro evaluation in 3D culture}

The seeding of PBCB matrices with fibrin gelimmobilized primary osteoblasts proved to be technically feasible. The gel immobilization provided reproducible and homogenous cell distribution within the construct and helped to minimize cell loss during seeding to a negligible level. Directly after seeding the pores of the matrix were homogenously filled with the fibrin gel. Osteoblasts were evenly distributed and spread in the network of fibrin fibrils within the first two weeks following seeding (Fig. 1A). After 7 days in vitro, osteoblasts started to adhere to the PBCB matrix (data not shown). After 14 days osteoblasts are attached to the matrix (Fig. 1B and 1C). At this time, osteoid formation and secretion of calcified extracellular matrix was evident at the cell-matrix interface on histological cross sections (Fig. 1C).

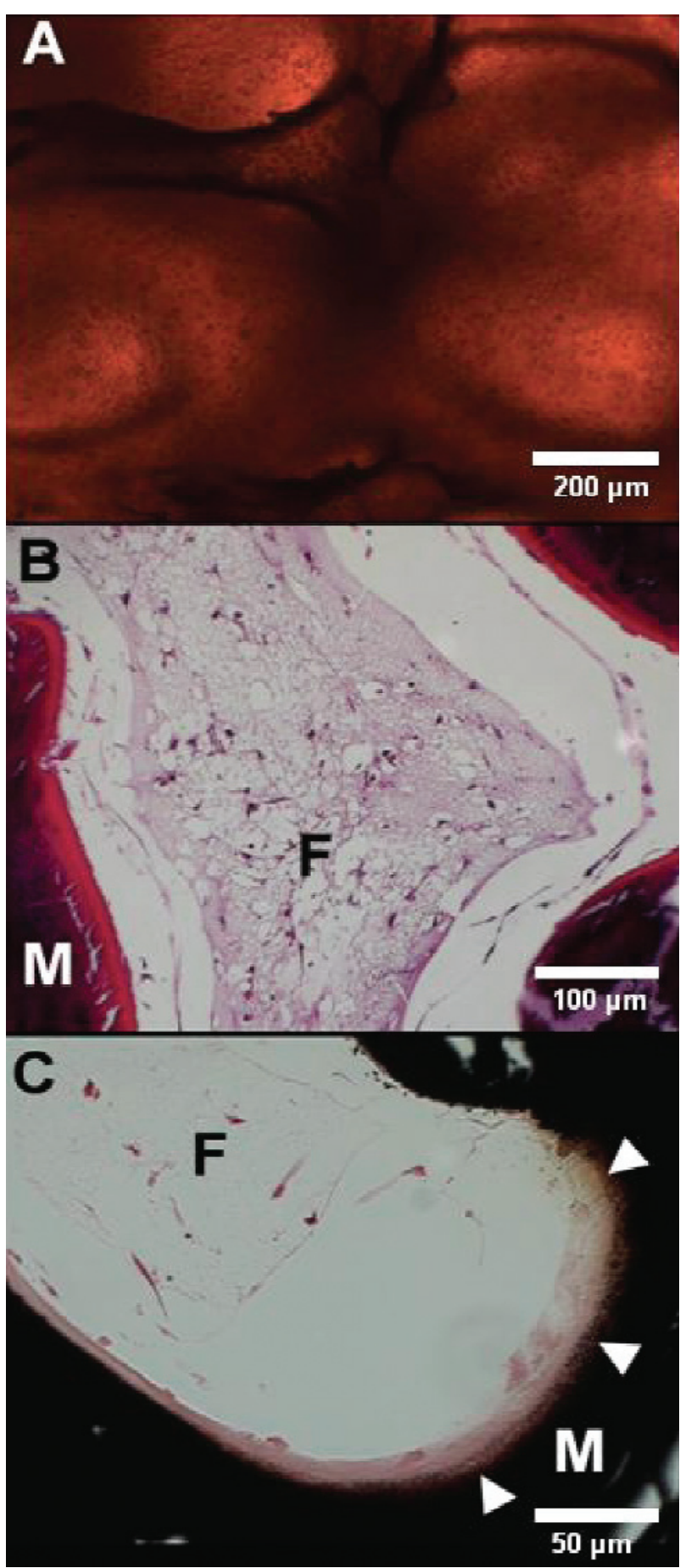

Fig. 1 Cell-seeded PBCB matrix prior to implantation (14 days in 3D culture). A: The pores of the matrix are filled with osteoblast-containing fibrin gel. (Magnification $x$ 100). B: The osteogenic cells are homogenously distributed within the fibrin gel (F). $\mathrm{M}=\mathrm{PBCB}$ matrix. (HE stain $x$ 200). C: Calcified extracellular matrix with granular structure (white arrow heads) is secreted by the fibrin gel-immobilized osteoblasts (F) and deposited to the PBCB matrix (homognously stained, M). Osteoblasts are attached to the PBCB matrix (Von Kossa stain x 400). 


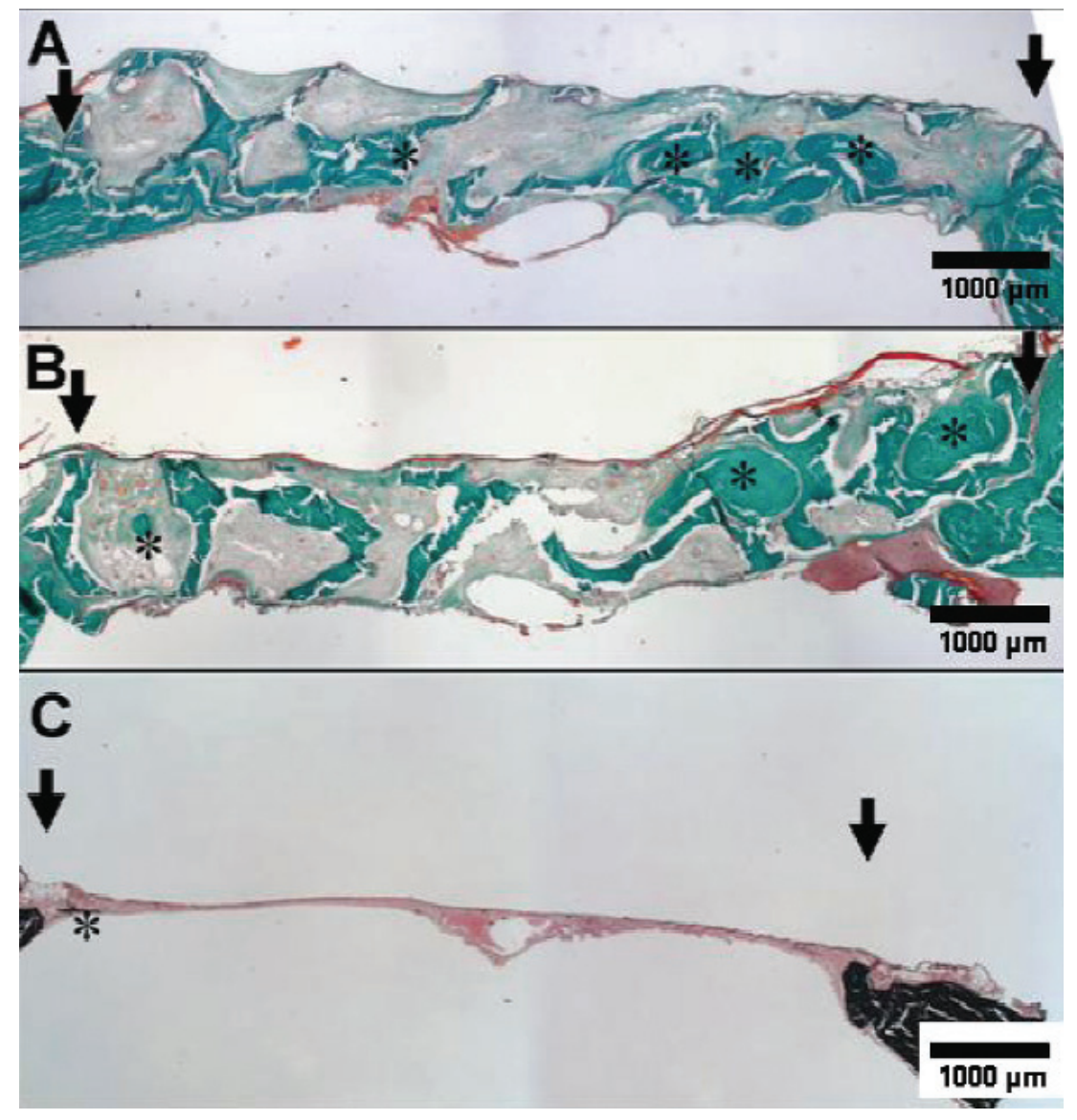

Fig. 2 Specimen from groups A-C at 4 months after surgery. Arrows indicate the defect's margins. A: Bone formation (asterisks) in contact to the $\mathrm{PBCB}$ matrix is evident in group A (PBCB matrix + osteoblasts). (Masson Goldner stain $\times 25)$. B: The pattern of bone formation (asterisks) in cell free PBCB matrices (group B) is comparable to cell-containing matrices from group A. (Masson-Goldner stain x 25). C: Untreated control defect (group C). There is virtually no bone formation within the created defect. (Von Kossa stain x 25).

\section{Surgeries and macroscopic aspects}

The number of animals per group reported in Table 1 reflects the number of included animals $(n=96)$. Five animals died shortly (within $12 \mathrm{hrs)} \mathrm{after} \mathrm{surgery} \mathrm{due}$ to surgical complications such as epidural hematoma. These animals were excluded from the study. The other animals tolerated the surgical procedure without significant complications. No infections or implantrelated complications were observed on clinical or histological observation. No extrusion of the implants occurred over the observation period. In groups A and $\mathrm{B}$, the constructs appeared macroscopically well integrated into the surrounding calvarial bone. Although biomechanical testing was not performed in this study the fixation was described as "mechanically stable" by the surgeon who performed the explantations. The constructs protected the underlying brain reliably and mechanically stabile at any time. In the control group $\mathrm{C}$ the non-reconstructed empty defects were filled with a fibrous layer of tissue. In these animals there were no macroscopic signs of bony regeneration of the defects.

\section{Histology}

At one month following surgery, in the control group C (empty defect) one animal displayed limited bony ingrowth at the periphery of the defect. Apart from this single animal there was no significant bone formation evident at any time (Fig. 2C). Fibrous tissue filled the defect sites in these animals. In groups A (PBCB matrix + osteoblasts) and B (PBCB matrix without osteoblasts) there was increasing multifocal bone formation evident between one and four months following implantation (Figs. 2A, 2B, $3 \mathrm{~A}$ and $3 \mathrm{~B})$. The implants were tightly anchored in the surrounding calvarial bone at the recipient site with bony contacts across the graft-calvarial interface (Fig. 3C). Formation of granulation tissue, generalized inflammation or encapsulation of the implants was not observed in any of these histological specimens. Beginning at two months after implantation, resorption of the matrix and formation of lacunae in presence of multinucleated cells, deposition of calcified 
extracellular matrix to the biomaterial and finally formation of new bone in direct contact to the PBCB matrix could be observed on histological cross sections from groups $\mathrm{A}$ and $\mathrm{B}$ (Figs. $4 \mathrm{~A}-\mathrm{E})$. The morphological appearance of the newly formed tissue within the PBCB matrix in groups $\mathrm{A}$ and $\mathrm{B}$ did not significantly change between 2 and 4 months following implantation. Fluorescent sequential labelling demonstrated bone formation in both groups with comparable rates (Fig. 4E shows bone formation in a matrix from the cell transplantation group A). Neovascularization occurred in cell-seeded and unseeded matrices with multiple evenly distributed capillaries branching throughout the entire scaffold architecture (Figs. 4A and 4B). Vascularization of the implants originated predominantly from the overlying soft tissues. The density of capillaries increased between 7 days and 2 months following implantation; however, this was not assessed quantitatively. Small islands of hematopoietic elements were evident in matrix pores and at the bone-biomaterial interface in association with bone formation only in implants from group A (PBCB matrix + osteoblasts) at 2 and 4 months following implantation (Fig. 4C and 4D). Histological analysis did not disclose significant morphological differences between cell-free and osteoblast-seeded PBCB matrices. However, bone formation in central regions of the implants was more pronounced in the cell-containing constructs from group A. Cartilaginous tissue was not evident at any time.

\section{CFDA labelling}

Short term analysis of implants seeded with CFDAlabelled osteoblasts demonstrated homogenous initial cell distribution. At days 0 and 1 after implantation, labelled vital osteoblasts were evident in high numbers in central and peripheral regions of the matrix (Fig. 5A). After day one, a dramatic decrease of osteoblast numbers within the constructs was observed. At day 7 after implantation some CFDA positive cells with physiologic morphology could be verified at the construct's periphery. Central regions of the matrix contained only very few viable osteoblasts (Fig. 5 B).

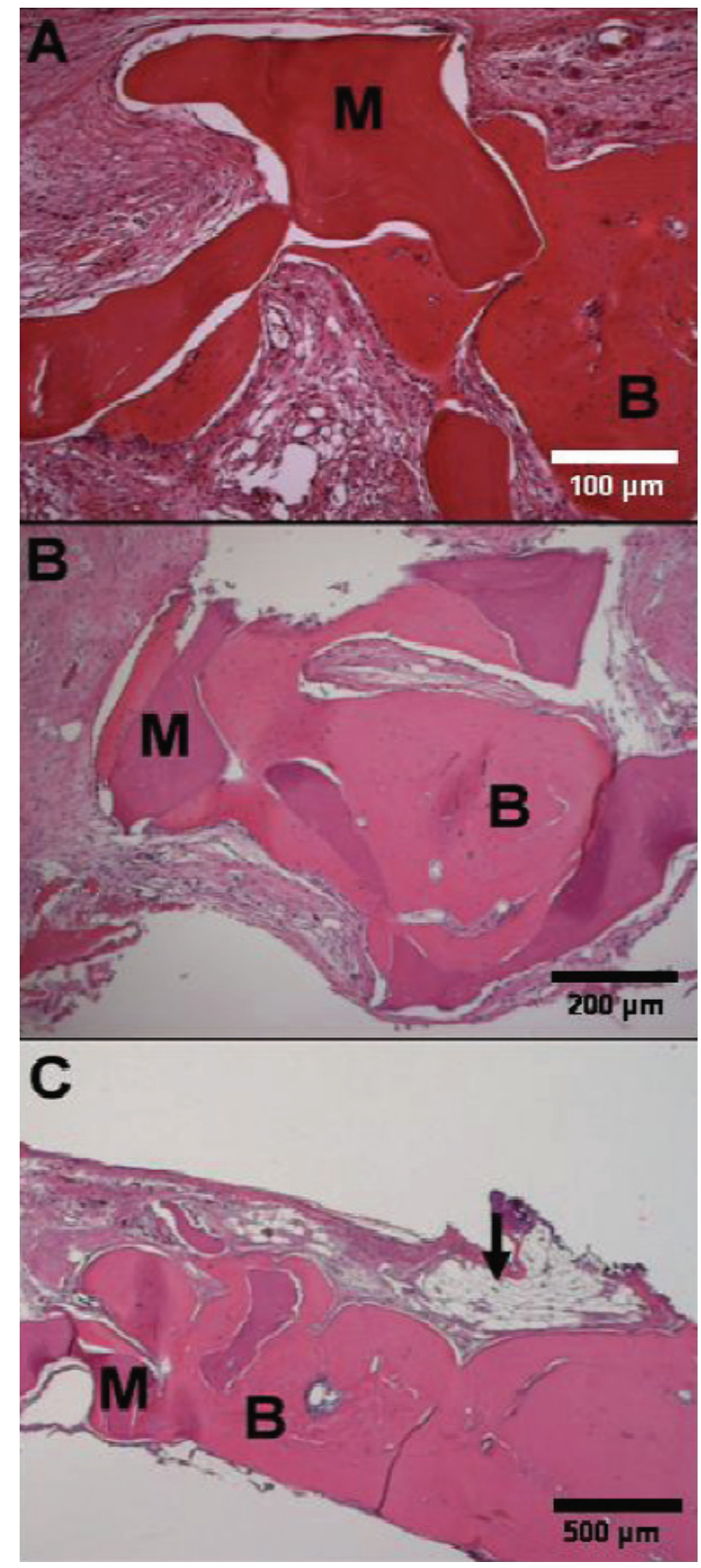

Fig. 3 Abundant appositional bone formation (B) in contact to the $\mathrm{PBCB}$ matrix $(\mathrm{M})$ at 4 months following implantation. The PBCB matrix stains darker and the void spaces of the former osteocytes that have been removed during processing are clearly evident. A: Cellfree matrix from group B (HE x 200). B: Osteoblastseeded matrix from group A (HE x 100). C: The PBCB matrix (M) is well integrated into the surrounding bone (B). Arrow indicates the margin of the critical size defect. (HE x 50). 

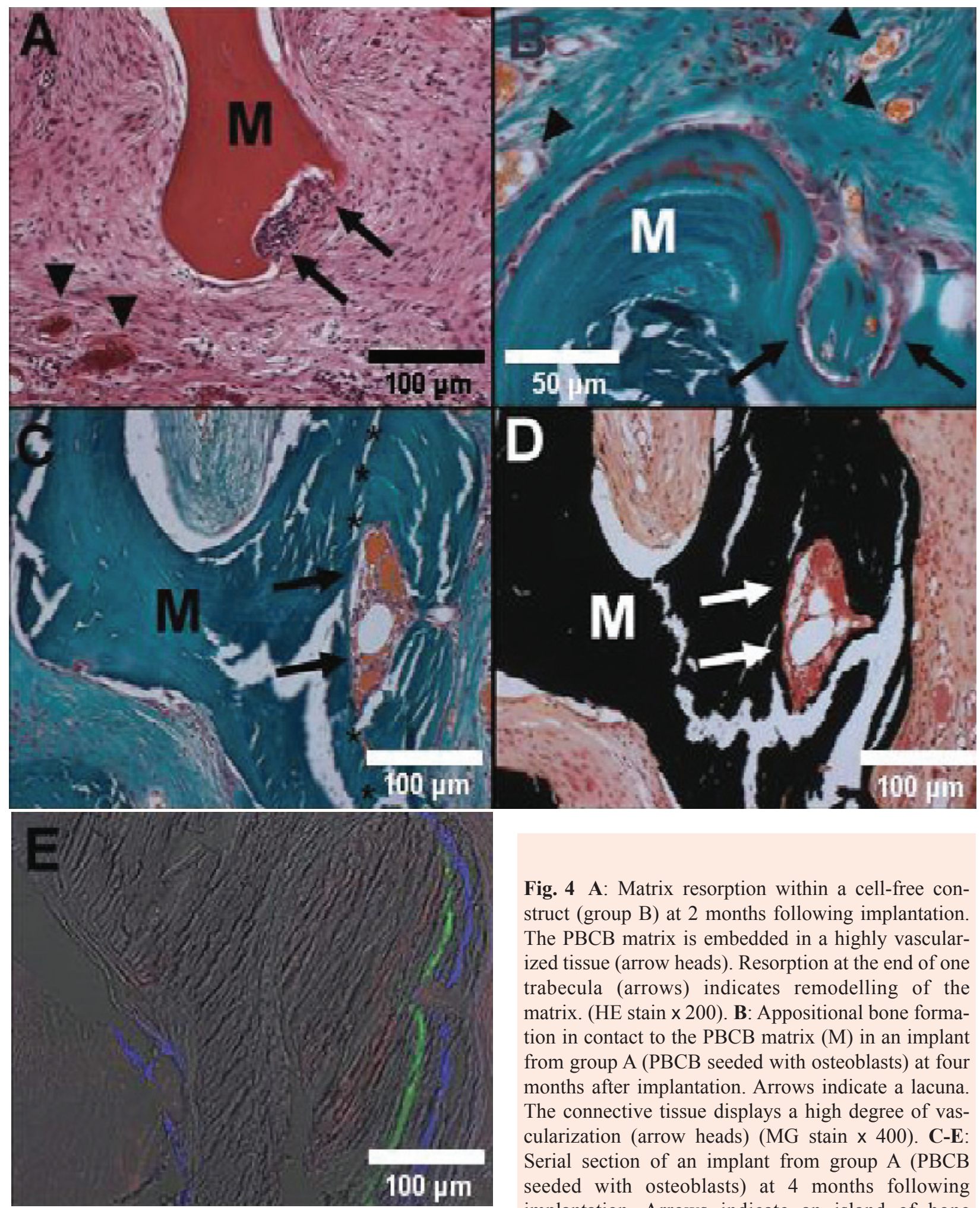

Fig. 4 A: Matrix resorption within a cell-free construct (group B) at 2 months following implantation. The PBCB matrix is embedded in a highly vascularized tissue (arrow heads). Resorption at the end of one trabecula (arrows) indicates remodelling of the matrix. (HE stain x 200). B: Appositional bone formation in contact to the PBCB matrix (M) in an implant from group A (PBCB seeded with osteoblasts) at four months after implantation. Arrows indicate a lacuna. The connective tissue displays a high degree of vascularization (arrow heads) (MG stain x 400). C-E: Serial section of an implant from group A (PBCB seeded with osteoblasts) at 4 months following implantation. Arrows indicate an island of bone marrow within the newly formed bone in contact to

the PBCB matrix (M). The margin of the PBCB matrix (M) is marked with asterisks (Fig. 4C). (C MG stain x 200, D Von Kossa stain $\times 200)$. E: Red, green and blue bands indicate active bone formation during the last month prior to explantation in a specimen from the cell transplantation group A. (polychrome fluorescent sequential labelling, confocal laser microscopy x 200). 


\section{Morphometric analysis}

The qualitative results from the histological analysis are reflected in the morphometric quantitative assessment of bone formation within the defects. In groups $\mathrm{A}$ (PBCB matrix + osteoblasts) and $\mathrm{B}$ (PBCB matrix alone) there was an increase in bone formation between one and four months. This increase was significant only in group B. (ratio bone area : total defect area in group A at 1 month $0.79 \pm 0.82$ $\%, 2$ months $1.66 \pm 2.45 \%$ and at 4 months $2.70 \pm$ $2.21 \%$. In group B at 1 month $0.51 \pm 0.68 \%$, at 2 months $2.99 \pm 3.54 \%$ and at 4 months $4.48 \pm 3.52$ $\%$. Group A p $<0.1$, group B $p<0.05$ ). In group C the amount of newly formed bone decreased over the observation period (group $\mathrm{C}$ at 1 month $0.63 \pm$ $1.66 \%, 2$ months $0.20 \pm 0.58 \%, 4$ months $0.09 \pm$ $0.25 \%$ ). At four months after transplantation the amount of newly formed bone in groups A and B was significantly larger than in the control group $\mathrm{C}$ $(\mathrm{p}<0.05)$. There was no statistically significant difference between groups A and B at any time (Fig. 6).

\section{Discussion}

Large calvarial bone defects are a therapeutic challenge in cranio- and maxillofacial surgery. In this study we evaluated a processed bovine cancellous bone matrix in a rat critical size calvarial defect model with regard to a possible clinical application in cranioplasty. Furthermore we aimed to enhance bone formation within this matrix by adding osteogenic cells to the construct prior to implantation. In the following, specific aspects of the study are discussed in detail.

\section{Fibrin gel immobilization of osteoblasts}

Fibrin gel-immobilization is a well established procedure for entrapment of different types of cells. Bensaid and co-workers reported successful culture and transplantation of human mesenchymal stem cells in a fibrin gel matrix [31]. Fibrin gel-immobilized osteoprogenitor cells have been successfully applied for reconstruction of critical sized nasal defects in a rat model [32]. The fibrin gel applied in the current study was a very low density gel (10

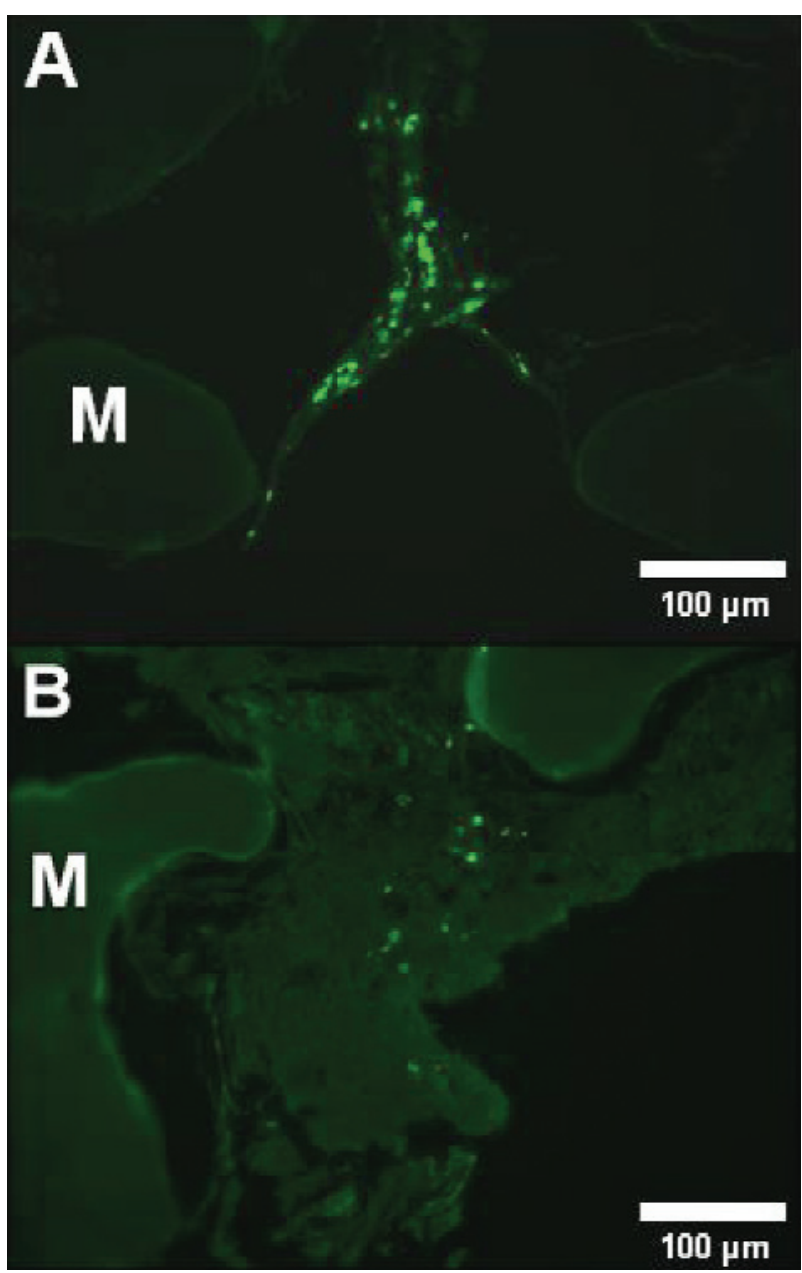

Fig. 5 CFDA labelling experiment. A: A significant number of morphologically intact osteoblasts are attached to the PBCB matrix (M) at day 1 following implantation (fluorescent microscopy x 200). B: After 7 days there is mainly condensed cell debris visible. There are virtually no intact labelled osteoblasts within the central parts of the construct. (fluorescent microscopy $\mathrm{x} 200$ ).

$\mathrm{mg} / \mathrm{ml}$ fibrinogen) which is resorbed within 7-14 days following subcutaneous implantation in rat models. Significant osteoconductive effects of this type of gel are not very likely since the matrix will disappear soon after implantation.

\section{In vitro evaluation}

Previously, we were able to demonstrate physiologic gene expression patterns in a detailed quantitative 


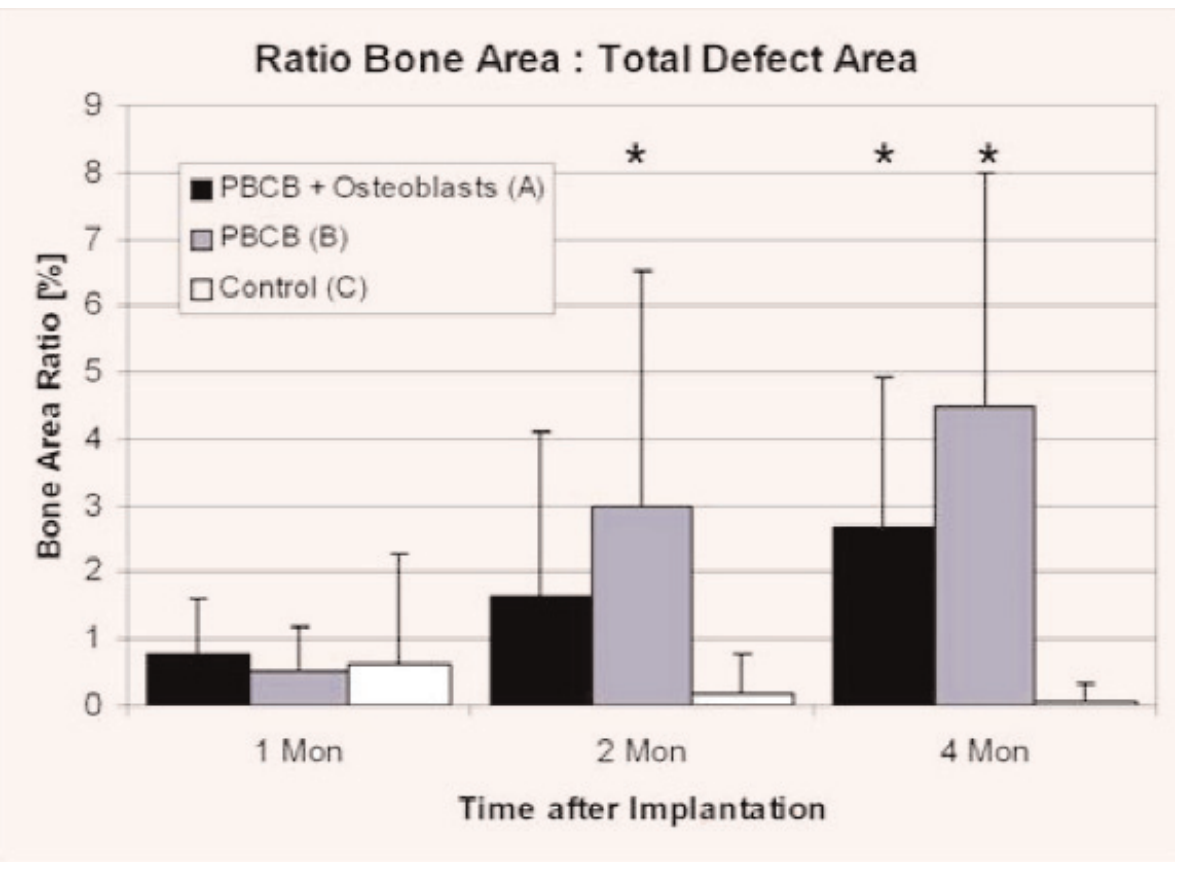

Fig. 5 Morphometric analysis of bone formation within the constructs. Group A: PBCB matrix + osteoblasts. Group B: PBCB matrix. Group C: untreated control defects. Bone formation is calculated as a ratio (area of newly formed bone : total defect area) and given as mean \pm standard deviation in \%. Each bar represents 8 individual samples. Asterisk indicates significant differences between experimental groups and control group C.

analysis of primary fibrin gel-immobilized rat osteoblasts in 3D culture in PBCB matrices [15]. In this study, osteoblasts displayed a physiological differentiated phenotype after 14 days in vitro following adequate osteogenic induction. In the current study histological evaluation demonstrated matrix secretion and calcification of the secreted matrix. These results suggest that the osteoblasts within the biomaterial display some aspects of differentiated cell function. This finding in combination with the above-mentioned experimental results suggests beneficial properties of PBCB matrices with regard to adhesion and differentiation of fibrin gel-immobilized osteoblasts in vitro.

\section{Animal model and assessment of bone formation}

The validity of the applied critical size defect model could be proven by the lack of bone formation in the untreated control group over the whole observation period. In contrast, in both experimental groups abundant bone formation was visible after 2 and 4 months following implantation.

There is a variety of different critical size defect models for evaluation of bone replacement systems [33]. Enhanced bone formation following implantation of cell-loaded biomaterials in calvarial criticalsize defects has been reported by many investigators
$[7,8]$. Some of these studies display considerable interindividual variance of the raw data. Many of the investigated matrices did not display superior osteoconductive capacities such as the PBCB matrix evaluated in our study, since the cell free matrices did not significantly enhance bone formation in comparison to the untreated control defects [8].

Histomorphometric analysis of bone formation within osteogenic implants is rather complex. "Defect closure" given in \% of the surface of the defect is a quite rough two-dimensional parameter that does not reflect the total amount of newly formed bone. Therefore we assessed the ratio of bone area : defect area in centrally located histological cross section as an indirect parameter for the volume of newly formed bone within the matrix. Although this parameter does not reflect the total amount of newly formed bone in 3D constructs the results of this morphometric analysis are comparable to the volume fraction of newly formed bone as assessed by micro $\mathrm{CT}$ in other experimental settings [7]. Due to the high radio opacity of the PBCB matrix, which is caused by a mineral content comparable to native bone, radiological studies such as CT or X-ray photographs did not provide reliable results in this study.

We observed a considerably high standard deviation of the morphometric data. The complex surgical setting and the small dimensions of the defects may 
interfere with efficient standardization of defect induction and matrix implantation. The high interindividual variance in combination with the osteoconductivity of the PBCB matrix and the low number of engrafted cells made it conceivably impossible to detect any significant differences between cell-free and cell-loaded matrices in the presented study. Heterotopic transplantation models exclude bone formation caused by osteoconductive material properties [34]. These models are therefore more sensitive for the detection of osteogenesis within biomaterials following implantation and may have the potential to demonstrate heterotopic bone formation within PBCB matrices in future experiments.

\section{Bone formation and matrix remodelling in cell-free PBCB matrices}

The presence of significant bone formation in the cell-free PBCB matrices (group B) in contrast to control group $\mathrm{C}$ clearly demonstrates the osteoconductive properties of this biomaterial. The matrix is integrated into the surrounding bone and a mechanically stabile fusion was observed at explantation in all specimens. The findings of bone formation and matrix resorption suggest that some remodelling takes place within the implants at 4 months after surgery. At the moment there are no reliable data on long term remodelling of this type of PBCB matrix in the rat model available. Based on the results of this study and preliminary experiments (unpublished data) we assume that it might take at least 12 months until significant matrix remodelling takes place in the applied model. Many clinical applications do not require truly osteogenic bone replacement. In these situations the cell-free PBCB matrix represents a reasonable therapeutic approach. The material has high initial mechanic stability and may even be fixed with screws or plates, if needed.

\section{Bone formation in cell-seeded PBCB matrices and cell engraftment}

To our disappointment, the osteoblasts-loaded PCBC matrices (group A) did not display enhanced bone formation in vivo when compared to the cellfree matrices from group B. The above-mentioned detailed in vitro study [15] demonstrated physiolog- ic osteoblastic differentiation and cell survival within PBCB matrices over a 14 days period. In addition, the CFDA labelling study demonstrates that the cells were viable upon implantation. Therefore direct negative (cytotoxic) influences of the matrix on the immobilized cells do not sufficiently explain the insufficient bone formation. However, within 7 days following implantation, the majority of the transplanted osteoblasts died as evidenced by nearly complete absence of CFDA-positive cells within the constructs.

Increased bone formation following implantation of osteoblast-seeded hard tissue matrices has been described by numerous investigators in different experimental settings. Several factors may influence bone formation in bioartificial bone tissues. The type of transplanted cell (osteoblast, mesenchymal stem cell, bone marrow stromal cell...), the specific surgical setting and last but not least the properties of the matrix material play an important role in any implantation model. The direct comparison of cell-seeded established biomaterials such as hydroxyl apatite and others with the PBCB matrix in an identical surgical setting may help to clarify the role of the matrix with regard to bone formation in osteogenic bioartificial bone tissues.

Another conceivable explanation for suboptimal bone formation in any implanted bioartificial bone tissue might be that there are considerable differences between the in vitro and initial in vivo environment. Although osteoblasts are nourished in vitro as well as initially in vivo by diffusion processes, the initial in vivo conditions may represent a challenge to the transplanted cells. Upon implantation, concentration of nutrients in the surrounding body fluid changes considerably. Furthermore, cells are confronted with initial inflammatory reaction. Exudate and fibrin are deposited to the matrix and present a barrier towards nutrients and oxygen. In addition, blood vessels start sprouting from the matrix' periphery to the center (extrinsic vascularization pattern) only upon implantation. In the initial phase following transplantation, cells within bioartificial tissues completely rely on diffusion processes for nutrition and oxygenation. Diffusion is limited to a 100 to $200 \mu \mathrm{m}$ distance from the surface of the implant [35]. Therefore upscaling of current tissue engineering concepts may be limited by inappropriate surface-to-volume ratios of large constructs. In future experiments we aim to investigate 
PBCB matrices in larger animal models with regard to implantation of tissue volumes suitable for clinical application in reconstructive surgery.

Incorporation of angiogenic growth factors or cultured endothelial cells may accelerate vascularization of the matrix and may consecutively enhance osteoblast survival [22, 36-38]. A different approach is the induction of vascularization prior to cell transplantation. Flap prefabrication strategies [39] or the implantation of a vascular carrier [40] allow generating prevascularized matrices for subsequent injection of osteogenic cells. These strategies are subject of current investigation in our laboratory [20]. The above-mentioned concepts may help to eventually increase transplantation efficiency and consecutive bone formation within cellseeded constructs.

\section{Conclusion}

This study demonstrates that processed bovine cancellous bone matrix (PBCB) represents a mechanically stabile, osteoconductive bone replacement material for applications in maxillofacial surgery. It is well integrated into bone defects and does not cause any foreign body reaction upon implantation. PBCB matrix supports osteoblast survival over a 14 days culture period and the cultured cells secret calcified matrix within the PBCB matrix upon induction of differentiation. Transplantation of primary osteoblasts within the PBCB matrix did not further increase bone formation in the presented experimental setting. Optimization of initial vascularization of the implants may enhance initial cell engraftment and may eventually allow the creation of a truly osteogenic biologic bone replacement system based on PBCB matrix.

\section{Acknowledgements}

The authors thank Mrs. Beate vom Hoevel and Dr. Yong Hong Zhang for expert technical assistance. Dr. M. Follo's help with the confocal laser microscope is highly appreciated. This work contains parts of L. Stangenberg's doctoral thesis. Prof. Dr. J. SchulteMönting's contribution to the statistical analysis
(ANOVA) is highly appreciated (Institute for Biomedical Statistics, University of Freiburg, Germany). This study was supported by grants from the German Federal Ministry of Education and Research (Grant Number 0312348) and from the Wissenschaftliche Gesellschaft Freiburg. The Tutobone ${ }^{\circledR}$ Matrices used in this study were supplied by Tutogen Medical Inc.

\section{References}

1. Younger EM, Chapman MW. Morbidity at bone graft donor sites. J Orthop Trauma. 1989; 3: 192-5.

2. Shin H, Jo S, Mikos AG. Biomimetic materials for tissue engineering. Biomaterials 2003; 24: 4353-64.

3. McAuliffe JA. Bone graft substitutes. J Hand Ther. 2003; 16: $180-7$.

4. Holy CE, Fialkov JA, Davies JE, Shoichet MS. Use of a biomimetic strategy to engineer bone. J Biomed Mater Res. 2003; 65A: 447-53.

5. Knaack D, Goad ME, Aiolova M, Rey C, Tofighi A, Chakravarthy P, Lee DD. Resorbable calcium phosphate bone substitute. J Biomed Mater Res. 1998; 43: 399-409.

6. Behravesh E, Zygourakis K, Mikos AG. Adhesion and migration of marrow-derived osteoblasts on injectable in situ crosslinkable poly(propylene fumarate-co-ethylene glycol)-based hydrogels with a covalently linked RGDS peptide. J Biomed Mater Res. 2003; 65A: 260-70.

7. Schantz JT, Hutmacher DW, Lam CX, Brinkmann M, Wong KM, Lim TC, Chou N, Guldberg RE, Teoh SH. Repair of calvarial defects with customised tissue-engineered bone grafts II. Evaluation of cellular efficiency and efficacy in vivo. Tissue Eng. 2003; 9 Suppl 1: S127-39.

8. Breitbart AS, Grande DA, Kessler R, Ryaby JT, Fitzsimmons RJ, Grant RT. Tissue engineered bone repair of calvarial defects using cultured periosteal cells. Plast Reconstr Surg. 1998; 101: 567-74.

9. Kadiyala S, Young RG, Thiede MA, Bruder SP. Culture expanded canine mesenchymal stem cells possess osteochondrogenic potential in vivo and in vitro. Cell Transplant. 1997; 6: 125-34.

10. Ohgushi H, Miyake J, Tateishi T. Mesenchymal stem cells and bioceramics: strategies to regenerate the skeleton. Novartis Found Symp. 2003; 249: 118-27.

11. Ignatius AA, Betz O, Augat P, Claes LE. In vivo investigations on composites made of resorbable ceramics and poly(lactide) used as bone graft substitutes. J Biomed Mater Res. 2001; 58: 701-9.

12. Cornu O, Banse X, Docquier PL, Luyckx S, Delloye C. Effect of freeze-drying and gamma irradiation on the mechanical properties of human cancellous bone. $J$ Orthop Res. 2000; 18: 426-31.

13. Hofmann C, Schadel-Hopfner M, Berns T, Sitter H, Gotzen L. [Influence of processing and sterilization on the mechanical properties of pins made from bovine cortical bone]. Unfallchirurg. 2003; 106: 478-82. 
14. Trentz OA, Hoerstrup SP, Sun LK, Bestmann L, Platz A, Trentz OL. Osteoblasts response to allogenic and xenogenic solvent dehydrated cancellous bone in vitro. Biomaterials 2003; 24: 3417-26.

15. Stangenberg L, Schaefer DJ, Buettner O, Ohnolz J, Moebest D, Stark GB, Horch RE, Kneser U. Differentiation of osteoblasts in 3D culture in processed cancellous bone matrix: A quantitative analysis of gene expression based on real-time RT-PCR. Tissue Eng. 2005; 11: 855-64

16. Schaefer DJ, Klemt C, Zhang XH, Stark GB. [Tissue engineering with mesenchymal stem cells for cartilage and bone regeneration]. Chirurg. 2000; 71: 1001-8.

17. Keith JD, Jr. Localized ridge augmentation with a block allograft followed by secondary implant placement: a case report. Int J Periodontics Restorative Dent. 2004; 24: 11-7.

18. Cancedda R, Mastrogiacomo M, Bianchi G, Derubeis A, Muraglia A, Quarto R. Bone marrow stromal cells and their use in regenerating bone. Novartis Found Symp. 2003; 249: 133-43.

19. Kon E, Muraglia A, Corsi A, Bianco P, Marcacci M, Martin I, Boyde A, Ruspantini I, Chistolini P, Rocca M, Giardino R, Cancedda R, Quarto R. Autologous bone marrow stromal cells loaded onto porous hydroxyapatite ceramic accelerate bone repair in critical-size defects of sheep long bones. J Biomed Mater Res. 2000; 49: 328-37.

20. Kneser U, Schaefer DJ, Polykandriotis E, Horch RE. Tissue Engineering of Bone: the Reconstructive Surgeon's Point of View. J Cell Mol Med. 2006; 10: 7-19.

21. Logeart-Avramoglou D, Anagnostou F, Bizios R, Petite H. Engineering bone: challenges and obstacles. J Cell Mol Med. 2005; 9: 72-84.

22. Tabata Y. Tissue regeneration based on growth factor release. Tissue Eng. 2003; 9: S5-15.

23. Lisignoli G, Zini N, Remiddi G, Piacentini A, Puggioli A, Trimarchi C, Fini M, Maraldi NM, Facchini A Basic fibroblast growth factor enhances in vitro mineralization of rat bone marrow stromal cells grown on nonwoven hyaluronic acid based polymer scaffold. Biomaterials. 2001; 22: 2095-105.

24. Kokubo S, Mochizuki M, Fukushima S, Ito T, Nozaki K, Iwai T, Takahashi K, Yokota S, Miyata K, Sasaki N. Long-term stability of bone tissues induced by an osteoinductive biomaterial, recombinant human bone morphogenetic protein-2 and a biodegradable carrier. Biomaterials 2004; 25: 1795-803.

25. Arnold U, Schweitzer S, Lindenhayn K, Perka C. Optimization of bone engineering by means of growth factors in a three-dimensional matrix. J Biomed Mater Res. 2003; 67A: 260-9.

26. Cheng SL, Lou J, Wright NM, Lai CF, Avioli LV, Riew KD. In vitro and in vivo induction of bone formation using a recombinant adenoviral vector carrying the human BMP-2 gene. Calcif Tissue Int. 2001; 68: 87-94.
27. Ohgushi H, Caplan AI. Stem cell technology and bioceramics: from cell to gene engineering. $J$ Biomed Mater Res. 1999; 48: 913-27.

28. Schmitz JP, Schwartz Z, Hollinger JO, Boyan BD. Characterization of rat calvarial nonunion defects. Acta Anat (Basel). 1990; 138: 185-92.

29. Maniatopoulos C, Sodek J, Melcher AH. Bone formation in vitro by stromal cells obtained from bone marrow of young adult rats. Cell Tissue Res. 1988; 254: 317-30.

30. Mueller-Stahl K, Kofidis T, Akhyari P, Wachsmann B, Lenz A, Boublik J, Heine M, Muehlfait V, Haverich A, Mertsching H. Carboxyfluorescein diacetate succinimidyl ester facilitates cell tracing and colocalization studies in bioartificial organ engineering. Int J Artif Organs. 2003; 26: 235-40.

31. Bensaid W, Triffitt JT, Blanchat C, Oudina K, Sedel L, Petite H. A biodegradable fibrin scaffold for mesenchymal stem cell transplantation. Biomaterials. 2003; 24 : 2497-502.

32. Tholpady SS, Schlosser R, Spotnitz W, Ogle RC, Lindsey WH. Repair of an osseous facial critical-size defect using augmented fibrin sealant. Laryngoscope 1999; 109: 1585-8.

33. Schmitz JP, Hollinger JO. The critical size defect as an experimental model for craniomandibulofacial nonunions. Clin.Orthop. 1986; 299-308.

34. Mauney JR, Jaquiery C, Volloch V, Heberer M, Martin I, Kaplan DL. In vitro and in vivo evaluation of differentially demineralized cancellous bone scaffolds combined with human bone marrow stromal cells for tissue engineering. Biomaterials 2005; 26: 3173-85.

35. Folkman J, Hochberg M. Self-regulation of growth in three dimensions. J Exp Med. 1973; 138: 745-53.

36. Peattie RA, Nayate AP, Firpo MA, Shelby J, Fisher RJ, Prestwich GD. Stimulation of in vivo angiogenesis by cytokine-loaded hyaluronic acid hydrogel implants. Biomaterials 2004; 25: 2789-98.

37. Wenger A, Stahl A, Weber H, Finkenzeller G, Augustin HG, Stark GB, Kneser U. Modulation of in vitro angiogenesis in a three-dimensional spheroidal coculture model for bone tissue engineering. Tissue Eng. 2004; 10: 1536-47.

38. Byrne AM, Bouchier-Hayes DJ, Harmey JH. Angiogenic and cell survival functions of vascular endothelial growth factor (VEGF). J Cell Mol Med. 2005; 9: 777-94.

39. Khouri RK, Upton J, Shaw WW. Principles of flap prefabrication. Clin Plast Surg. 1992; 19: 763-71.

40. Tanaka Y, Sung KC, Tsutsumi A, Ohba S, Ueda K, Morrison WA. Tissue engineering skin flaps: which vascular carrier, arteriovenous shunt loop or arteriovenous bundle, has more potential for angiogenesis and tissue generation? Plast Reconstr Surg. 2003; 112: 1636-44. 